\title{
Blocking MET receptor signaling in multiple myeloma cells in vitro and in vivo
}

\author{
Artur Jurczyszyn 1, A-F, Anna Zebzda 2, A-F, Joanna Gdula-Argasińska ${ }^{3, D, F}$, Jacek Czepiele ${ }^{4, E, F}$, \\ David H. Vesole ${ }^{5,6, E, F}$, William Perucki ${ }^{7, D}$, Marcin Majka ${ }^{2, A-F}$ \\ ${ }^{1}$ Department of Hematology, Faculty of Medicine, Jagiellonian University Medical College, Kraków, Poland \\ 2 Department of Transplantology, Faculty of Medicine, Jagiellonian University Medical College, Kraków, Poland \\ ${ }^{3}$ Radioligand Department, Faculty of Pharmacy, Jagiellonian University Medical College, Kraków, Poland \\ ${ }^{4}$ Department of Infectious Diseases, Faculty of Medicine, Jagiellonian University Medical College, Kraków, Poland \\ ${ }^{5}$ John Theurer Cancer Center, Hackensack University Medical Center, USA \\ ${ }^{6}$ School of Medicine, Georgetown University, USA \\ ${ }^{7}$ John Dempsey Hospital, Department of Medicine, University of Connecticut, Farmington, USA \\ A - research concept and design; $B$ - collection and/or assembly of data; $C$ - data analysis and interpretation; \\ $\mathrm{D}$ - writing the article; $\mathrm{E}$ - critical revision of the article; $\mathrm{F}$ - final approval of the article
}

Address for correspondence

Artur Jurczyszyn

E-mail: mmjurczy@cyf-kr.edu.pl

Funding sources

None declared

Conflict of interest

None declared

Received on February 14, 2016

Reviewed on July 13,2016

Accepted on January 4, 2017

\section{Abstract}

Background. Numerous studies have shown a role of the hepatocyte growth factor (HGF) as a ligand for the MET receptor in promoting aggressiveness in myeloma cells.

Objectives. The aim of this study was to confirm the presence of the MET receptor in myeloma cell lines, to establish a stable lentiviral construct directed against MET receptor mRNA and then to evaluate the effect of blocking MET receptor expression both in vitro and in vivo.

Material and methods. The U266 and INA6 cells were transduced using a lentiviral vector carrying siRNA to achieve the reduction of MET receptor expression. The ocular sinus of NOD/SCID mice was injected with wt-U266, shMET-U266 and shLacZ-U266 cells.

Results. MET receptor expression was demonstrated in all tested myeloma cell lines. Blocking the HGF/MET axis did not affect the growth of transduced U266 and INA6 cell lines. The inoculation of NOD/SCID mice with myeloma cells with reduced expression of MET led to increased survival of the animals.

Conclusions. MET receptor expression was constituently expressed in all tested myeloma cell lines. A lentiviral construct can effectively reduce the expression of the MET receptor in myeloma cells. Further studies are necessary to evaluate the effect of the reduction of MET receptor expression in multiple myeloma, focusing on animal models with a larger test group size.

Key words: hepatocyte growth factor, transduction, U266, INA6

DOI

$10.17219 /$ acem/68271

Copyright

Copyright by Author(s)

This is an article distributed under the terms of the

Creative Commons Attribution Non-Commercial License

(http://creativecommons.org/licenses/by-nc-nd/4.0/) 
Multiple myeloma (MM) is a plasma cell malignancy characterized by uncontrolled proliferation of clonal plasma cells manifested clinically by increased secretion of monoclonal immunoglobulins or light chains. Over the past decade, new therapeutic agents have been incorporated into the treatment algorithm for patients with MM, increasing their survival rates. ${ }^{1-3}$ Further research into new treatments for MM is essential in order to improve the survival of patients. One potential target for MM therapy is the hepatocyte growth factor (HGF)/mesenchymal-epithelial transition (MET) axis. ${ }^{4}$

HGF belongs to the plasminogen protein family. Under physiological conditions, HGF can be produced by fibroblasts, fat collecting liver cells, bone marrow stromal cells, endothelial, and epithelial cells. HGF is the only known ligand of the MET receptor. The MET receptor is a tyrosine kinase receptor. It is composed of an extracellular 50 $\mathrm{kD} \alpha$ chain and a transmembrane $140 \mathrm{kD} \beta$ chain. HGF binding to the MET receptor results in tyrosine phosphorylation of the c-terminal portion, leading to the recruitment of adaptive and signaling proteins, and the activation of multiple signal transduction pathways. This activation results in the migration, mitosis and morphogenesis of multiple cell lines, and excessive activation has been implicated in the pathogenesis of many cancers. ${ }^{5,6}$

In patients with MM who showed elevated levels of $\mathrm{HGF}$, there is a direct correlation between serum HGF levels and aggressiveness of the disease. ${ }^{4,7}$ The prognosis of patients with MM is usually worse in the presence of high levels of HGF. ${ }^{8-10}$ Among the many factors activating myeloma cells, HGF is one of the main factors and the presence of the MET receptor is a common finding in myeloma cells, both at the mRNA and protein levels. ${ }^{11,12} \mathrm{HGF}$ is secreted by cells of the bone marrow stroma, thus allowing for autocrine and paracrine regulation of tumor cells, stimulates proliferation and inhibits apoptosis of myeloma cells. ${ }^{11} \mathrm{HGF}$-induced IL-11 secretion from osteoblastic cells may contribute to osteolysis seen in a majority of patients with MM. ${ }^{13}$

Numerous studies have shown a role of HGF/MET axis in promoting aggressiveness in myeloma cells. The aim of our study was to confirm the presence of the MET receptor in myeloma cell lines and to establish a stable lentiviral construct directed against MET receptor mRNA. An additional goal was to determine whether silencing of the MET receptor would have an impact on the growth of myeloma cells in vitro and life expectancy of mice inoculated with transduced myeloma cells in vivo.

\section{Material and methods}

\section{Myeloma cell lines}

Myeloma cell lines INA6 and U266 (American Type Culture Collection, Manassas, USA) were cultured in RPMI 1640 medium (Gibco BRL, ThermoFisher Scientific, Waltham,
USA) and supplemented with fetal bovine serum (FBS, 10\% and 15\%, respectively) (PAA Laboratories, GE Healthcare Bio-Sciences Austria GmbH, Pasching, Austria), $2 \mathrm{mmol}$ L-glutamine, and $100 \mathrm{IU} / \mathrm{mL}$ penicillin and $10 \mu \mathrm{g} / \mathrm{mL}$ streptomycin (Gibco BRL), at $37^{\circ} \mathrm{C}$ in an atmosphere of $5 \%$ $\mathrm{CO}_{2}$ with $95 \%$ humidity. Additionally, INA6 required the presence of IL- 6 at the concentration of $2 \mathrm{ng} / \mathrm{mL}$.

\section{Assessment of gene expression by real-time polymerase chain reaction}

RNA was isolated using RNeasy Mini Kit (Qiagen, Valencia, USA). The concentration and purity of the obtained RNA was assessed by measuring absorbance at a wavelength of 260 and $280 \mathrm{~nm}$, using a DU 640B spectrophotometer (Beckman Coulter, Fullerton, USA). RNA was transcribed into cDNA using MMLV reverse transcriptase (Promega, Madison, USA) and non-specific primers, called random primers, (Promega). The analysis of gene expression was performed by quantitative PCR in real time (qRT-PCR) based on specific TaqMan probes (Applied Biosystems, Foster City, USA), using an ABI PRISM 7300 Sequence Detection System (Applied Biosystems). The components of the reaction mixture used in the qRT-PCR were: TaqMan PCR Master Mix $25 \mu \mathrm{L}$, cDNA $100 \mathrm{ng}$, $20 \times$ probe $2.5 \mu \mathrm{L}$, water added until final volume was $50 \mu \mathrm{L}$. Probes used for qRT-PCR were manufactured by Applied Biosystems accordingly: TaqMan MET Hs01565589_m1 and GAPDH Hs99999905_m1. To calculate results, we analyzed relative gene expression using a $\Delta \Delta \mathrm{Ct}$ calculation, based on the comparison values of $\mathrm{Ct}$ for the test and control gene. We used mRNA isolated from umbilical cord blood mononuclear cells as a negative control.

\section{Determination of the MET receptor presence}

Immunohistochemical staining was used to determine the presence of the MET receptor by means of cytospin preparations. Preparations were stained using monoclonal anti-MET antibodies (1:100 dilution) visualized with the DAKO LSAB2 visualization system and kit (DakoCytomation, Glostrup, Denmark). In order to visualize the antigenantibody reaction, 1 drop of streptavidin was added, and then chromogen was used as an activating agent. The obtained preparations were stained with hematoxylin and embedded in glycerol gel. Samples were evaluated using a light microscope produced by Olympus (Tokyo, Japan).

\section{Transfection of myeloma cell lines U266 and INA6}

Separate test tubes were prepared according to the following specifications: Lipofectamine 2000 in a solution of $50 \mathrm{~mL}$ OptiMEM I medium (Invitrogen, ThermoFisher Scientific, Waltham, USA) and $1 \mu \mathrm{g}$ siRNA in $50 \mathrm{~mL}$ 
OptiMEM I medium without FBS or antibiotics. These agents were combined and allowed to stand for $20 \mathrm{~min}$ to form 2000 siRNA-lipofectamine complexes.

Cell lines U266 and INA6 were transduced using a lentiviral vector carrying siRNA directed against MET to achieve the reduction of gene expression, and LacZ as a control to confirm proper action of the receptor. The lentiviral vector used for the experiments was based on a 19-nucleotide sequence of siRNA directed against MET 5'-CCG AGA AGU AUG UGA UGA ATT-3'. Cell lines U266 and INA6 were seeded at a concentration of $3 \times 104$, and converted using lentiviral transduction. Ten $\mu \mathrm{L}$ viral particles suspended in $1 \mathrm{~mL}$ RPMI with 10\% FBS was added to the cells in the presence of $6 \mathrm{mg} / \mathrm{mL}$ hexadimethrine bromide (Sigma-Aldrich Co., St. Louis, USA). After $48 \mathrm{~h}$, blasticidin was introduced $(10 \mu \mathrm{g} / \mathrm{mL}$ for the U266 line and $4 \mu \mathrm{g} / \mathrm{mL}$ for the INA6 line). After 12 days, dead or apoptotic MM cells not resistant to blasticidin were observed. We established a naming convention for these cells by adding wt (wild type, input cell lines), shMET or shLacZ for the transduced cell lines. Transduced and wild type cell lines U266 and INA6 cells were plated in complete RPMI medium onto 6-well culture plates at a concentration of $2 \times 104 /$ well, and then counted in specified time intervals to judge growth. The number of cells after $24 \mathrm{~h}$ was used as the baseline and compared to the number of cells after 48, 72 and 96 h of incubation.

\section{Analysis of a mute MET receptor in a mouse model using transduced U266 cell lines}

For in vivo studies, 15 non-obese diabetic/severe combined immunodeficiency (NOD/SCID) mice purchased from Jackson Laboratories, Bar Harbor, USA were used. This study was approved by the local ethics committee. The NOD/SCID mice were irradiated at 300 cGy using a GammaCell irradiator. The mice were divided into 3 subgroups, 5 in each group, and after $24 \mathrm{~h}$, the ocular sinus was injected with $5 \times 106$ cells of the following cell lines: wt-U266, shMET-U266 and shLacZ-U266. After the death of the mice, their long bones were isolated, fixed in paraformaldehyde, and then prepared in paraffin blocks for slide mounting immunohistochemical staining.

\section{Statistical analysis}

Statistical analysis of the results was performed using GraphPad Prism 4.02 (GraphPad Software Inc., La Jolla, USA). Survival analysis of the mice was performed using the Kaplan-Meier analysis. The statistical significance of differences between groups was tested using the Student's t-test or one-way ANOVA at a significance level of $\mathrm{p}<0.05$.

\section{Results}

We observed MET receptor expression in myeloma cell lines both at the mRNA and protein levels. In order to investigate the presence of MET receptor mRNA in myeloma cell lines, 3 independent experiments were performed using RT-PCR. There was no presence of MET receptor expression in blood mononuclear cells isolated from the umbilical cord as a negative control (Fig. 1).

\begin{tabular}{|c|c|c|c|}
\hline Line & Control cells & U266 & INA6 \\
\hline MET expression & - & + & + \\
\hline
\end{tabular}
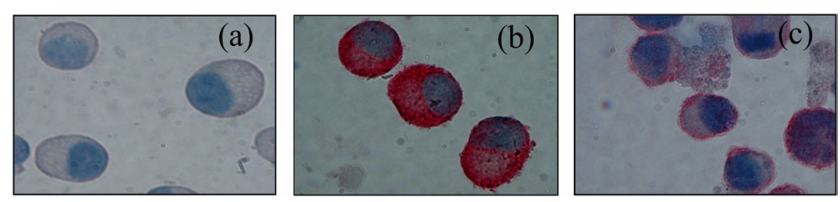

Fig. 1. A - immunohistochemical staining of multiple myeloma cell lines for the presence of the MET receptor; B - (a) control cells, (b) U266 cells, (c) INA6 cells. Representative pictures, microscope magnification $\times 200$

\section{Characteristics of transduced U266 and INA6 myeloma cell lines}

The line shMET-U266 showed a significant decrease in mRNA expression for the MET gene compared to wt-U266 (0.38 vs $8.63 \mathrm{au}$ ), and shMET-INA6 showed a significant decrease in mRNA expression for the $M E T$ gene compared to wt-INA6 (0.36 vs $6.0 \mathrm{au}$ ) (Fig. 2A). The mRNA expression for the MET gene in shLacZ-U266/INA6 confirms proper acting of the lentiviral construct. The decrease in MET mRNA was reflected in decreased expression of the MET receptor (Fig. 2B). Western blotting detected a significant decrease in the amount of protein compared to MET

A
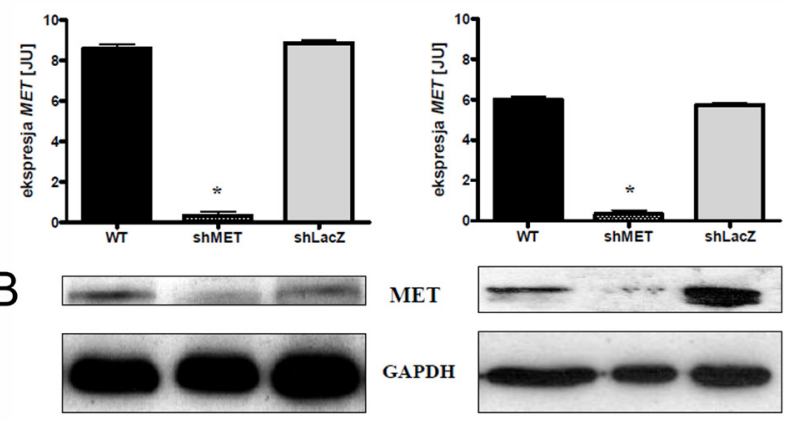

MET

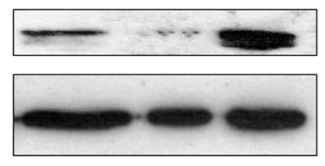

Fig. 2. shMET-U266 showed a significant decrease in mRNA expression for the MET gene compared to wt-U266 (0.38 vs $8.63 \mathrm{au}$ ), and shMET-INA6 showed a significant decrease in MRNA expression for the MET gene compared to wt-INA6 (0.36 vs $6.0 \mathrm{au}$ )

A - mRNA expression for the MET gene in shLacZ-U266/INA6 confirms proper acting of the lentiviral construct; $B$ - the decrease in MET mRNA was reflected in decreased expression of the MET receptor. 


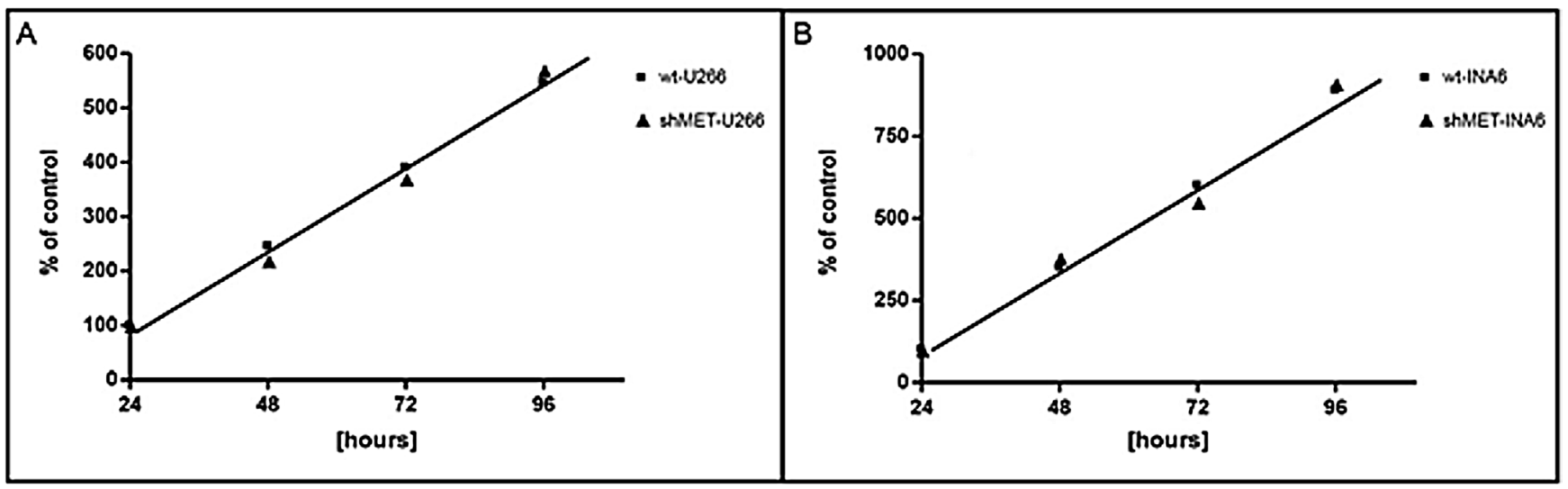

Fig. 3. Comparison of the growth rate of wild type cells and cells transduced with a lentiviral vector

A - comparison of cell lines wt-U266 and shMET-U266; B - comparison of lines wt-INA6 and shMET-INA6.

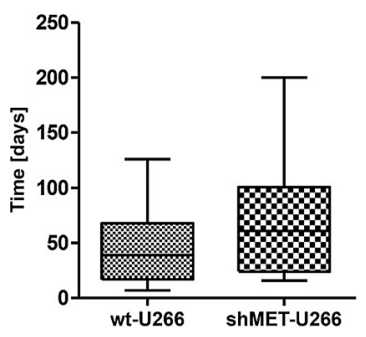

Fig. 4. Effect of reducing the expression of MET in U266 cells on the survival of NOD/SCID mice

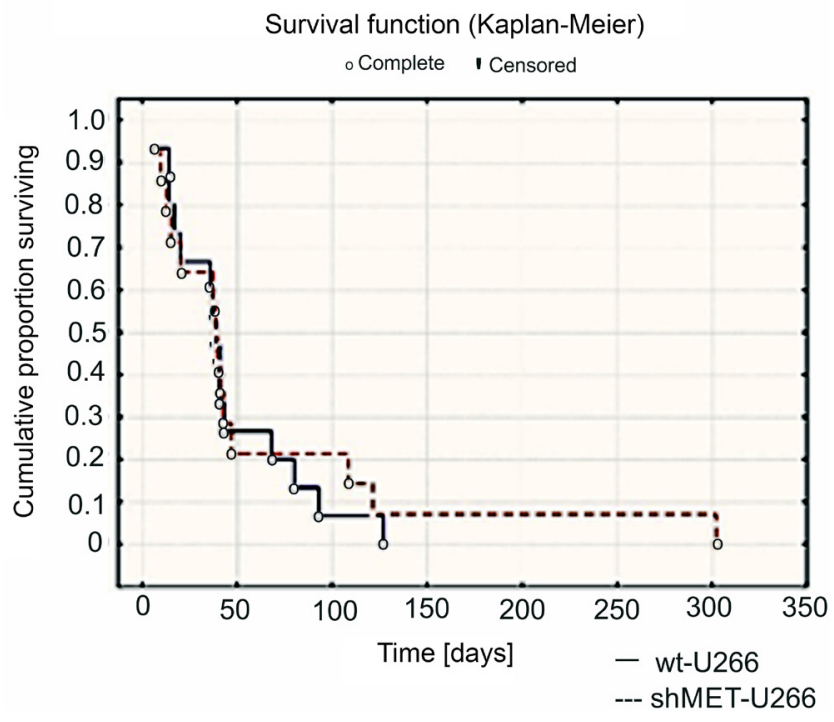

Fig. 5. Survival expectancy of NOD/SCID mice - murine myeloma model

output lines. The lentiviral construct directed against MET receptor mRNAs, can effectively reduce the MET receptor.

Blocking the HGF/MET axis did not affect the growth of cell lines U266 and INA6.

There was no difference in growth between wt-U266 and shMET-U266, or between wt-INA6 and shMET-INA6 (Fig. 3). Median survival rates were as follows: wt-U266 - 40 days, shMET-U266 - 62 days (Fig. 4). Blocking the HGF/MET axis increased the survival of NOD/SCID mice, but the observed changes were not statistically significant (Fig. 5).

\section{Discussion}

The HGF/MET axis is involved in many physiological processes including growth, repression of intercellular adhesion, cell migration, epithelial-mesenchymal transition (EMT), inhibition of apoptosis, proliferation and morphogenesis as well as in wound healing and tissue regeneration..$^{5,714-16}$ This axis is also important in oncogenesis and plays a significant role in the migration, proliferation and adhesion of various neoplastic cells. Overexpression or excessive activation of HGF/MET has been demonstrated in mesenchymal and epithelial tumors such as breast cancer, ovarian cancer, gastrointestinal tract cancer, lung cancer, prostate cancer, glioblastoma, sarcomas, and MM. ${ }^{17}$ MET is also partly responsible for the metastasis of tumor cells by increasing their migration, secretion of proteolytic enzymes, ability to survive in the blood vessels, and ability to remain in the capillary bed. Together with other mitogenic factors, such as the SDF-1-CXCR4 axis, it is involved in the colonization of distant tissues by tumor cells, and stimulates their growth in microenvironments normally foreign to them. ${ }^{4}$

In this study, we observed the expression of MET mRNA and MET protein in myeloma cell lines. Reports on the role of HGF/MET axis encourage research on the use of inhibitors of this axis in the treatment of various neoplastic diseases, including multiple myeloma. With respect to the methods of inhibiting the axis of HGF/MET, one could consider inhibiting the attachment of HGF to the MET receptor, the prevention of dimerization of the MET receptor, the inhibition of the MET receptor tyrosine kinase, or the inhibition of the expression of HGF or MET. ${ }^{17}$ Blocking the interaction between the receptor and the ligand may occur after the application of competitive antagonists of HGF or antibodies directed against HGF or MET. NK4 is a truncated form of HGF and competes with it for binding at the MET receptor, and it does 
not have the effect of receptor activation. In a study by Brockmann et al., it was demonstrated that NK4 inhibits glioblastoma growth in mice with implanted tumor cells via pro-apoptotic and anti-mitogenic mechanisms. ${ }^{17} \mathrm{NK} 4$ exhibited anti-cancer effects mainly due to the inhibition of invasion and metastasis as well as the inhibition of angiogenesis-dependent tumor growth. ${ }^{18}$ Du et al. showed reduced growth of myeloma cells in mice treated with NK4. ${ }^{19}$ Martens et al. demonstrated growth inhibition using anti-HGF neutralizing antibodies in a mouse model of glioblastoma. ${ }^{20}$ Vigna et al. also applied anti-MET antibodies, which resulted in the inhibition of the growth of epithelial cancers. ${ }^{21}$ Recent studies have demonstrated that the use of angiotensin IV analog, norleual as an antagonist of HGF/MET, not only blocked its dimerization, but also inhibited HGF-dependent MET activation and had anticancer activity. ${ }^{22}$ The semaphorin domain is necessary for MET receptor dimerization. Kong-Beltran et al. demonstrated that recombinant soluble semaphorin domains block phosphorylation of the MET receptor, regardless of the presence or absence of HGF. ${ }^{23}$ To inhibit the MET tyrosine kinase and its signaling activity, low molecular weight inhibitors, such as geldanamycin, K252a, indolinone, and its analogs (17-AAG, 17-DMAG) or PHA665752 were used. ${ }^{25-28}$ Hov et al., using PHA665752 small molecule acting directly on the receptor MET, observed the inhibition of cell proliferation, migration and adhesion in ANBL6 myeloma cell lines. ${ }^{24}$ To reduce the expression of MET and HGF, nonsense RNA or ribozymes can be used. Nonsense RNA or single-stranded DNA is a molecule consisting of a 15-25 nucleotide sequence, which can be used to induce degradation of mRNA or block its translation. Blocking the $M E T$ gene causes a decrease in expression of the MET receptor, thus inhibiting the growth of tumor cells. ${ }^{26}$ Abounader et al. demonstrated that ribozymes, naturally occurring RNA molecules, can catalyze the specific cleavage of mRNA and reduce the expression of HGF and MET, resulting in the inhibition of activation of the MET receptor, and consequently the inhibition of colony formation and migration of tumor cells in vitro. ${ }^{28}$ Que et al. revealed that the down-regulation of MET inhibits the proliferation and invasion of U266 myeloma cells, and increases their chemosensitivity to doxorubicin and bortezomib. ${ }^{29-31}$

Posttranscriptional gene silencing, a technique that is based on the phenomenon of RNA interference (RNAi) through siRNA, uses the natural process of gene expression dependent on double-stranded RNA. Translation of the mRNA is blocked by the introduction of siRNA with a sequence complementary to the target RNA. The use of an adenoviral vector encoding siRNA against MET in gastric cancer, prostate cancer and glioma cell lines resulted in decreased mitogenic activity. MET gene silencing resulted in the induction of apoptosis. ${ }^{32,33}$ In vitro siRNA can be delivered directly to the cell or in the form of vectors expressing siRNA. ${ }^{34,35}$ The use of viral systems allows for long-term silencing of the receptor and high reproducibility of results.

In our study, in order to reduce the expression of mRNA for MET, a lentiviral model was used. Transduction of U266 and INA6 cells using siRNA test vectors effectively silenced the expression of MET at both the mRNA and protein levels. Taulli et al. examined rhabdomyosarcoma cells transduced with an siRNA lentiviral vector directed against the MET receptor, and observed not only inhibition of migration, but also decreased cell proliferation. ${ }^{36}$ In our study, we observed that the transduced U266 and INA6 cell lines showed no differences in the rate of in vitro growth compared to the wt. Differences previously reported probably occurred due to the use of different experimental designs and different tumor types. Taulli et al. used a lentiviral vector in rhabdomyosarcoma cell lines induced by the presence of doxycycline in assessing the impact of the MET receptor inhibition over 5 days of RNAi induction. ${ }^{36}$ In the present study, we used myeloma cell lines constitutively reduced in the expression of the MET receptor, and experiments were performed at least 3 weeks after the introduction of the vector. Hypothetically, prolonged exposure to siRNA may allow cells to develop a mechanism to avoid apoptosis.

The present study evaluated whether blocking the HGF/MET axis using lentiviral vectors may be a potential therapy to treat multiple myeloma. HGF is a potent chemoattractant for cells expressing the MET receptor on their surface. As a result of the increasing gradient of HGF, bloodborne myeloma cells settle in the bone marrow. ${ }^{37}$ A study by Teoh et al. demonstrated in U266 cells cocultured with mesenchymal stromal cells transfected with IL-6 siRNA a significant inhibition of cell growth, IL-6 synthesis, and suggested potential use of RNA interference-mediated therapy for multiple myeloma. ${ }^{38}$ Impaired response to the increased HGF gradient, caused by the reduction of the expression of the MET receptor, may influence the ability of tumor cells to migrate toward the bone marrow, which could hypothetically decrease neoplastic aggressiveness. Our experiments demonstrate that mice inoculated with myeloma cells with reduced expression of MET were characterized by higher median survival time than animals treated with wild type myeloma cell lines. The observed changes were not statistically significant; however, it should be noted that the test group of mice was not particularly large, and the observed difference in number of survival days, 40 vs 62 , was evident. This gives us hope that a larger study group could lead to statistically significant results.

In conclusion, MET receptor expression is typical for myeloma cell lines. The lentiviral construct directed against the MET receptor mRNAs can effectively reduce expression of the MET receptor in myeloma cells. Further studies are necessary to evaluate the effect of the reduction of MET receptor expression in multiple myeloma, focusing on animal models with a larger test group size. 


\section{References}

1. Ludwig $H$, Sonneveld $P$, Davies F, et al. European perspective on multiple myeloma treatment strategies in 2014. Oncologist. 2014;19;829_ 844.

2. Warren JL, Harlan LC, Stevens J, Little RF, Abel GA. Multiple myeloma treatment transformed: A population-based study of changes in initial management approaches in the United States. J Clin Oncol. 2013;31:1984-1989.

3. Kumar SK, Dispenzieri A, Lacy MQ, Gertz MA, Buadi FK. Continued improvement in survival in multiple myeloma: Changes in early mortality and outcomes in older patients. Leukemia. 2014;28:1122-1128.

4. Gambella M, Palumbo A, Rocci A. MET/HGF pathway in multiple myeloma: From diagnosis to targeted therapy? Expert Rev MolDiagn. 2015;15(7):881-893.

5. Kato T, Oka K, Nakamura T, Ito A. Decreased expression of MET during differentiation in rat lung. Eur J Histochem. 2016;60:2575. doi:10.4081/ ejh.2016.2575.

6. Jia Y, Dai G, Wang J, et al. c-MET inhibition enhances the response of the colorectal cancer cells to irradiation in vitro and in vivo. Oncol Lett. 2016;11:2879-2885.

7. Ferrucci A, Moschetta M, Frassanito MA, et al. A HGF/cMET autocrine loop is operative in multiple myeloma bone marrow endothelial cells and may represent a novel therapeutic target. Clin Cancer Res. 2014:20:5796-5807.

8. Jakob C, Sterz J, Zavrski I, et al. Angiogenesis in multiple myeloma. Eur J Cancer. 2006;42:1581-1590.

9. Boissinot M, Vilaine M, Hermouet $S$. The hepatocyte growth factor (HGF)/Met axis: A neglected target in the treatment of chronic myeloproliferative neoplasms? Cancers (Basel). 2014;6(3):1631-1669.

10. Wader KF, Fagerli UM, Holt RU, Børset M, Sundan A, Waage A. Soluble c-Met in serum of patients with multiple myeloma: Correlation with clinical parameters. Eur J Haematol. 2011; 87:394-399.

11. Mahtouk K, Tjin EP, Spaargaren M, Pals ST. The HGF/MET pathway as target for the treatment of multiple myeloma and B-cell lymphomas. Biochim Biophys Acta. 2010;1806:208-219.

12. Kristensen IB, Christensen JH, Lyng MB, et al. Hepatocyte growth factor pathway upregulation in the bone marrow microenvironment in multiple myeloma is associated with lytic bone disease. Br J Haematol. 2013;161(3):373-382.

13. Petrini I. Biology of MET: A double life between normal tissue repair and tumor progression. Ann Transl Med. 2015;3:82.

14. Sakai K, Aoki S, Matsumoto K. Hepatocyte growth factor and MET in drug discovery. J Biochem. 2015;157(5):271-284.

15. Watanabe K, Hirata M, Tominari T, et al. The MET/VEGFR-targeted tyrosine kinase inhibitor attenuates FMS-dependent osteoclast differentiation and bone destruction induced by prostate cancer. J Biol Chem. 2016. doi:jbc.M116.727875

16. Robinson KW, Sandler AB. The role of MET receptor tyrosine kinase in non-small cell lung cancer and clinical development of targeted anti-MET agents. Oncologist. 2013;18(2):115-122.

17. Brockmann MA, Papadimitriou A, Brandt M, Fillbrandt R, Westphal M, Lamszus K. Inhibition of intracerebral glioblastoma growth by local treatment with the scatter factor/hepatocyte growth factor-antagonist NK4. Clin Cancer Res. 2003;9(12):4578-4585.

18. Nakamura T, Sakai K, Nakamura T, Matsumoto K. Anti-cancer approach with NK4: Bivalent action and mechanisms. Anticancer Agents Med Chem. 2010;10(1):36-46.

19. Du W, Hattori Y, Yamada T, et al. NK4, an antagonist of hepatocyte growth factor (HGF), inhibits growth of multiple myeloma cells: Molecular targeting of angiogenic growth factor. Blood. 2007;109:3042-3049.
20. Martens T, Schmidt NO, Eckerich C, et al. A novel one-armed anti-cMet antibody inhibits glioblastoma growth in vivo. Clin Cancer Res. 2006:12:6144-6152.

21. Vigna E, Pacchiana G, Mazzone M, et al. "Active" cancer immunotherapy by anti-Met antibody gene transfer. Cancer Res. 2008;68:9176-9183.

22. Kawas LH, Yamamoto BJ, Wright JW, Harding JW. Mimics of the dimerization domain of hepatocyte growth factor exhibit anti-Met and anticancer activity. J Pharmacol Exp Ther. 2011;339(2):509-518.

23. Kong-Beltran M, Stamos J, Wickramasinghe D. The Sema domain of MET is necessary for receptor dimerization and activation. Cancer Cell. 2004;6:75-84.

24. Hov $\mathrm{H}$, Holt RU, Rø TB, et al. A selective c-Met inhibitor blocks an autocrine hepatocyte growth factor growth loop in ANBL-6 cells and prevents migration and adhesion of myeloma cells. Clin Cancer Res. 2004;10:6686-6694.

25. Christensen JG, Burrows J, Salgia R. c-Met as a target for human cancer and characterization of inhibitors for therapeutic intervention. Cancer Letters. 2005;225:1-26.

26. Christensen JG, Schreck R, Burrows J, et al. A selective small molecule inhibitor of c-Met kinase inhibits c-Met-dependent phenotypes in vitro and exhibits cytoreductive antitumor activity in vivo. Cancer Res. 2003;63:7345-7355.

27. Stabile LP, Lyker JS, Huang L, Siegfried JM. Inhibition of human nonsmall cell lung tumors by a c-Met antisense/U6 expression plasmid strategy. Gene Ther. 2004;11:325-335.

28. Abounader R, Lal B, Luddy C, et al. In vivo targeting of SF/HGF and c-met expression via U1snRNA/ribozymes inhibits glioma growth and angiogenesis and promotes apoptosis. FASEB J. 2002;16:108-110.

29. Que W, Chen J, Chuang M, Jiang D. Knockdown of c-Met enhances sensitivity to bortezomib in human multiple myeloma U266 cells via inhibiting Akt/mTOR activity. APMIS. 2012;120:195-203.

30. Que W, Chen J. Knockdown of c-Met inhibits cell proliferation and invasion and increases chemosensitivity to doxorubicin in human multiple myeloma U266 cells in vitro. Mol Med Rep. 2011;4:343-349.

31. Shen A, Wang $L$, Huang $M$, et al. c-Myc alterations confer therapeutic response and acquired resistance to c-Met inhibitors in MET-addicted cancers. Cancer Res. 2015;75(21):4548-4559.

32. Phan LM, Fuentes-Mattei E, Wu W, et al. Hepatocyte growth factor/ CMET pathway activation enhances cancer hallmarks in adrenocortical carcinoma. Cancer Res. 2015;75(19):4131-4142.

33. Elbashir SM, Harborth J, Weber K, TuschI T. Analysis of gene function in somatic mammalian cells using small interfering RNAs. Methods. 2002;26:199-213.

34. Yi Y, Kim HJ, Mi P, et al. Targeted systemic delivery of siRNA to cervical cancer model using cyclic RGD-installed unimer polyion complexassembled gold nanoparticles. J Control Release. 2016;28;244(Part B): 247-256. doi: 10.1016/j.jconrel.2016.08.041

35. Liu J, Xue H, Zhang J, et al. MicroRNA-144 inhibits the metastasis of gastric cancer by targeting MET expression. J Exp Clin Cancer Res. 2015;17:34-35

36. Taulli R, Scuoppo C, Bersani F, et al. Validation of MET as a therapeutic target in alveolar and embryonal rhabdomyosarcoma. Cancer Res. 2006;66:4742-4749.

37. Jankowski K, Kucia M, Wysoczynski M, et al. Both hepatocyte growth factor (HGF) and stromal-derived factor-1 regulate the metastatic behavior of human rhabdomyosarcoma cells, but only HGF enhances their resistance to radiochemotherapy. Cancer Res. 2003;63:79267935.

38. Teoh HK, Chong PP, Abdullah M, et al. Small interfering RNA silencing of interleukin- 6 in mesenchymal stromal cells inhibits multiple myeloma cell growth. Leuk Res. 2016;40:44-53. 